\title{
Frozen Blastocyst Transfer during in Vitro Fertilization/Intracytoplasmic Sperm Injection Cycle Lowers the Rate of Ectopic Pregnancy
}

\author{
Weijie Xing, Liuhong Cai, Li Sun, Jianping Ou* \\ Center for Reproductive Medicine, The Third Affiliated Hospital of Sun Yat-sen University, Guangzhou, China \\ Email: *oujianping1968@qq.com
}

How to cite this paper: Xing, W.J., Cai, L.H., Sun, L. and Ou, J.P. (2017) Frozen Blastocyst Transfer during in Vitro Fertilization/Intracytoplasmic Sperm Injection Cycle Lowers the Rate of Ectopic Pregnancy. Open Journal of Obstetrics and Gynecology, 7, 1183-1188.

https://doi.org/10.4236/ojog.2017.712120

Received: October 24, 2017

Accepted: November 12, 2017

Published: November 15, 2017

Copyright $\odot 2017$ by authors and Scientific Research Publishing Inc. This work is licensed under the Creative Commons Attribution International License (CC BY 4.0).

http://creativecommons.org/licenses/by/4.0/

\begin{abstract}
This study aimed to determine whether frozen blastocyst transfer can lower the incidence rate of ectopic pregnancy. A total of 1577 in vitro fertilization/ intra-cytoplasmic cycles were analyzed, including 757 fresh D3-ET cycles, 429 frozen D3-ET cycles, and 391 frozen D5-ET cycles. The differences of ages, the proportion of IVF cycles, the proportion of primary infertility, and the proportion of tubal factor between three groups were not significant. The clinical pregnancy rate in Frozen D5-ET Group was 62.15\%, which was significantly higher than other two groups $(46.10 \% ; 38.93 \% ; P<0.05)$. The miscarriage rates of three groups were similar. The ectopic pregnancy rate in Frozen D5-ET Group was $0.41 \%$, which was significantly lower than that in Frozen D3-ET Group $(2.99 \%, P<0.05)$ and slightly lower than that in Fresh D3-ET Group $(2.29 \%, P=0.089)$. Results from this study show that the transfer of frozen D5 blastocysts can significantly decrease the incidence rate of ectopic pregnancy.
\end{abstract}

\section{Keywords}

Fresh Embryo Transfer, Blastocyst Transfer, Ectopic Pregnancy

\section{Introduction}

Ectopic pregnancy occurs when an embryo implants outside of the uterine cavity, and can be fatal if rupture or bleeding presents. In in vitro fertilization and embryo transfer (IVF-ET), the Day 3 or Day 5 embryo is transplanted directly into the uterine cavity using a tube, which should theoretically prevent ectopic 
pregnancy, but in fact increases its occurrence. Ectopic pregnancy is one of the main complications of IVF-ET, with an occurrence rate of $2 \%-5 \%$, compared with $1 \%$ in natural pregnancies [1] [2].

Studies have shown that the transplanted embryo does not immediately implant in the uterine cavity [3]. Instead, it first swims to the oviducts before returning to the uterine cavity. If this process is affected, ectopic pregnancy may result. Controlled ovarian hyperstimulation $(\mathrm{COH})$ is used during IVF-ET; this technique causes an imbalance in estrogen and progesterone during embryo implantation, affecting endometrial receptivity and oviduct peristalsis, and possibly accounting for the increase in ectopic pregnancy [4] [5]. Frozen blastocyst transfer, however, is performed several months after superovulation, thus avoiding the effects of $\mathrm{COH}$ on the endometrium and oviducts, and possibly lowering the occurrence rate of ectopic pregnancy [4].

Many studies have investigated the relationship between frozen blastocyst transfer and ectopic pregnancy, but a consensus has yet to be reached. Huang et al. conducted a retrospective study of clinical outcomes in over 30,000 IVF cycles, and reported that frozen blastocyst transfer can significantly decrease the risk of ectopic pregnancy; however, a separate study conducted in Belgium over the course of a decade reported no significant differences in ectopic pregnancy risk between IVF-ET and frozen blastocyst transfer. Other studies analyzed data from single or small numbers of reproductive medical centers, and did not arrive at a consensus.

This paper is a retrospective analysis of clinical data on fresh and frozen blastocyst transfer cycles collected in our clinic between September 2015 and March 2017, to determine whether frozen blastocyst transfer can lower the incidence rate of ectopic pregnancy.

\section{Materials and Methods}

This study retrospectively analyzed 757 cycles of fresh D3 embryo transfer, 429 cycles of frozen D3 blastocyst transfer, and 391 cycles of frozen D5 blastocyst transfer from September 2015 to August 2017. This retrospective study was approved by the Third Affiliated Hospital of Sun Yat-sen University Medicine Ethics Committee. Patient records and information were anonymized and deidentified prior to analysis. This was a non-interventional, retrospective, single-center cohort study of patients in a routine clinical practice. To reflect the broad range of patients typically encountered in this clinical practice, no inclusion/exclusion criteria were applied to the baseline characteristics.

Agonadotropin releasing hormone agonist (GnRH-a) long protocol oran antagonist protocol were used for $\mathrm{COH}$. When $\geq 2$ dominant follicles measured at least $18 \mathrm{~mm}$ in diameter, or $\geq 4$ dominant follicles measured at least $16 \mathrm{~mm}$ in diameter, 6000 - 10,000 U of human chorionic gonadotropin (hCG) was injected, and eggs were removed $36 \mathrm{~h}$ after injection. Fertilization was performed through normal IVF or intracytoplasmic sperm injection (ICSI), and fresh blas- 
tocyst transfer was performed after $72 \mathrm{~h}$. The embryos were frozen-thawed by vitrification.

Before frozen blastocyst transfer, the endometrium was prepared using hormone replacement therapy. Ultrasound examination was performed 2 - 5 days following onset of menstruation, and a 2-mg twice daily oral dose of estradiol valerate was started (Progynova, Schering AG, Germany); the dose was increased to $3 \mathrm{mg}$ twice daily on day 5 , and continued for 5 days. Another B scan was performed 10 days after drug administration; when the endometrium reached $8 \mathrm{~mm}$ or more, $40 \mathrm{mg} / \mathrm{d}$ injections of progesterone were initiated. D5 blastocysts were transferred 6 days after initial progesterone treatment.

At 12 - 14 days following embryo transfer, patients with a positive urine test were designated as biochemical pregnancy. Blood hCG levels were measured, and ultrasound performed 3 weeks later was used as a marker of clinical pregnancy. If the B scan reflected a failure to implant in the uterine cavity, patients were designated as having ectopic pregnancies. Miscarriages were defined as losses prior to 28 weeks.

SPSS13.0 software was used to perform statistical analysis. Data were represented as mean \pm standard deviation. Comparison between the two groups was analyzed with ONE WAY ANOVA. Enumeration data were analyzed with chi-square test, with $P<0.05$ representing statistical significance.

\section{Results}

A total of 1577 IVF/ICSI cycles were analyzed, including 757 fresh D3-ET cycles, 429 frozen D3-ET cycles, and 391 frozen D5-ET cycles. From Table 1, we found that the differences of ages, the proportion of IVF cycles, the proportion of primary infertility, and the proportion of tubal factor between three groups were not significant. The number of transferred embryos in Frozen D3-ET Group was $2.07 \pm 0.44$, which was significantly more than that in other two groups $(1.98 \pm$ $0.3 ; 1.65 \pm 0.48 ; P<0.05)$.

Table 1. The clinical materials and outcomes in three groups.

\begin{tabular}{cccc}
\hline Variables & $\begin{array}{c}\text { Fresh D3-ET } \\
(\mathrm{N}=757)\end{array}$ & $\begin{array}{c}\text { Frozen D3-ET } \\
(\mathrm{N}=429)\end{array}$ & $\begin{array}{c}\text { Frozen D5-ET } \\
(\mathrm{N}=391)\end{array}$ \\
\hline Age & $33.99 \pm 5.53$ & $33.22 \pm 5.91$ & $32.85 \pm 4.49$ \\
Primary infertility (n, \%) & $38.97 \%$ & $36.60 \%$ & $33.25 \%$ \\
No. of IVF cycles (n, \%) & $82.17 \%$ & $81.35 \%$ & $81.07 \%$ \\
Tubal factor (n, \%) & $46.10 \%$ & $41.72 \%$ & $44.87 \%$ \\
Thickness of endometrium & $11.31 \pm 7.48^{*}$ & $9.97 \pm 1.97$ & $10.43 \pm 6.79$ \\
No. of embryos transferred & $1.98 \pm 0.3^{*}$ & $2.07 \pm 0.44^{*}$ & $1.65 \pm 0.48^{*}$ \\
Miscarriage rate & $11.46 \%$ & $13.77 \%$ & $10.70 \%$ \\
Clinical pregnancy rate & $46.10 \%$ & $38.93 \%$ & $62.15 \%{ }^{*}$ \\
Ectopic pregnancy rate & $2.29 \%$ & $2.99 \%$ & $0.41 \%{ }^{*}$ \\
\hline
\end{tabular}

Note: ${ }^{\star}$ means $P<0.05$ versus other two groups. 
The differences of clinical pregnancy rate in three groups were significant. The clinical pregnancy rate in Frozen D5-ET Group was 62.15\%, which was significantly higher than other two groups $(46.10 \% ; 38.93 \% ; P<0.05)$. The miscarriage rates of three groups were similar.

The ectopic pregnancy rate in Frozen D5-ET Group was $0.41 \%$, which was significantly lower than that in Frozen D3-ET Group $(2.99 \%, P<0.05)$ and slightly lower than that in Fresh D3-ET Group $(2.29 \%, P=0.089)$.

\section{Discussion}

Ectopic pregnancy is one of the most serious complications in assisted reproductive technology, and reducing its occurrence is a major objective in reproductive medicine. The results presented in this study suggest that with D3 embryos, the incidence rate of ectopic pregnancy remains similar regardless of whether fresh or frozen blastocyst transfer is used. A subgroup analysis on frozen embryo transfer cycles, however, showed that the incidence rate of ectopic pregnancy with D5 embryos is significantly lower than that with D3 embryos $(P<0.05)$.

The cause of ectopic pregnancy during IVF/ICSI remains unclear, but previous literature suggests a number of major possibilities:

1) Occurrence may be linked to controlled ovarian hyperstimulation. Under normal conditions, progesterone decreases uterine contraction during the luteal phase. In $\mathrm{COH}$, however, estradiol $\left(\mathrm{E}_{2}\right)$ levels are abnormally high, resulting in a hormonal imbalance (high estrogen and low progesterone), affecting both oviduct peristalsis and embryo motility, and causing ectopic pregnancy [6] [7]. Wang et al. analyzed incidence rates of ectopic pregnancy in polycystic ovary syndrome patients receiving IVF treatment, and reported that when $\mathrm{E}_{2}$ levels exceed $4085 \mathrm{pg} / \mathrm{mL}$, ectopic pregnancy occurs at a rate of 3.4\%; when $\mathrm{E}_{2} \leq 4085$ $\mathrm{pg} / \mathrm{mL}$, the rate of ectopic pregnancy decreases significantly to $2.0 \%$. In addition, abnormal increases in $\mathrm{E}_{2}$ levels cause abnormal uterine activities and contractions, which are also important contributing factors for the occurrence of ectopic pregnancy [8] [9]. Studies by Clayton et al. and Shapiro et al. showed that when hormone levels exceed physiological thresholds, the risk of ectopic pregnancy increased [4] [10].

2) Pathological changes in the oviduct caused by inflammation, adhesions, and surgery may constitute major risk factors for ectopic pregnancy following IVF-ET [11] [12]. A period of 3 - 5 days is required for implantation following IVF-ET. Prior to implantation, the embryo often appears to be in a freely-moving state. A change in this characteristic determines the likelihood that the embryo will move towards the oviducts and begin its development. Any degree of damage to the oviducts can directly lead to a decrease in cilia and limited peristalsis, which may consequently hinder the transport of the embryo to the uterus, resulting in ectopic pregnancy as the stalled embryo begins its development in the oviducts. A meta-analysis by Zhang et al. in 2016 reported that the transfer of D5 embryos resulting in a lower risk of ectopic pregnancy than the transfer of D3 
embryos may be attributed to the fact that transplanted D5 embryos have a shorter period of motility than D3 embryos do. This decreases the effects of oviduct abnormalities on freely swimming blastocysts, and therefore the risk of ectopic pregnancy [13]. This is consistent with our findings, which reflect a lower incidence rate of ectopic pregnancy in frozen blastocyst transfer cycles with D5 blastocysts.

3) Some studies have suggested racial factors in ectopic pregnancy, as well as factors related to $\mathrm{COH}$ procedures, IVF techniques and other methodology, including the location of transplant tube tip, injection volume, etc. These have been shown to be strongly correlated with the occurrence of ectopic pregnancy following IVF, but there is no consensus regarding this matter.

Ectopic pregnancy is a common complication of IVF-ET, which both decreases the rate of live births and subjects the patient to the associated danger of rupture and bleeding or adverse effects from conservative treatment. As a result, investigating methods to decrease the incidence rate is of critical importance. Results from our study suggest that the incidence rate of ectopic pregnancy with D5 embryos is significantly lower than that with D3 embryos. The frozen blastocyst transfer cycles included in this study are hormone replacement therapy cycles, and take into account the effects of endometrial factors and hormonal levels. These results may be attributed to a shorter pre-implantation period in the transfer of D5 blastocysts compared with that of D3 blastocysts, which lowers the effects of oviduct abnormalities to the free-swimming embryo, and therefore the risk of ectopic pregnancy. However, our sample size was limited, and our results will need to be supported by further proof from large-scale randomized controlled trials.

\section{Conclusion}

Results from this study show that the transfer of frozen D5 blastocysts can significantly decrease the incidence rate of ectopic pregnancy. This provides clinicians with a basis for reference. Prioritizing D5 blastocysts may lower the incidence rate of ectopic pregnancy and increase the live birth rate.

\section{References}

[1] Farquhar, C.M. (2005) Ectopic Pregnancy. The Lancet, 366, 583-591. https://doi.org/10.1016/S0140-6736(05)67103-6

[2] Fang, C., Huang, R., Wei, L.N. and Jia, L. (2015) Frozen-Thawed Day 5 Blastocyst Transfer Is Associated with a Lower Risk of Ectopic Pregnancy than Day 3 Transfer and Fresh Transfer. Fertility and Sterility, 103, 655e-661e.

[3] Polyzos, N.P. and Devroey, P. (2012) Significantly Lower Ectopic Pregnancy Rates after Frozen Embryo Transfer: Implications toward Segmentation of in Vitro Fertilization Treatment. Fertility and Sterility, 98, 1419-1420. https://doi.org/10.1016/j.fertnstert.2012.08.044

[4] Shapiro, B.S., Daneshmand, S.T., De Leon, L., Garner, F.C., Aguirre, M. and Hudson, C. (2012) Frozen-Thawed Embryo Transfer Is Associated with a Significantly 
Reduced Incidence of Ectopic Pregnancy. Fertility and Sterility, 98, 1490-1494. https://doi.org/10.1016/j.fertnstert.2012.07.1136

[5] Fernandez, H., Coste, J. and Job-Spira, N. (1991) Controlled Ovarian Hyperstimulation as a Risk Factor for Ectopic Pregnancy. Obstetrics and Gynaecology, 78, 656-659.

[6] Hoover, R.N., Hyer, M., Pfeiffer, R.M., Adam, E., Bond, B., Cheville, A.L., et al. (2011) Adverse Health Outcomes in Women Exposed in Utero to Diethylstilbestrol. The New England Journal of Medicine, 365, 1304-1314. https://doi.org/10.1056/NEJMoa1013961

[7] Wang, J., Wei, Y., Diao, F., Cui, Y., Mao, Y., Wang, W., et al. (2013) The Association between Polycystic Ovary Syndrome and Ectopic Pregnancy after in Vitro Fertilization and Embryo Transfer. American Journal of Obstetrics and Gynaecology, 209, 139.e1-139.e9.

[8] Lyons, R.A., Saridogan, E. and Djahanbakhch, O. (2006) The Reproductive Significance of Human Fallopian Tube Cilia. Human Reproduction Update, 12, 363-372. https://doi.org/10.1093/humupd/dml012

[9] Wanggren, K., Stavreus-Evers, A., Olsson, C., Andersson, E. and Gemzell-Danielsson, K. (2008) Regulation of Muscular Contractions in the Human Fallopian Tube through Prostaglandins and Progestagens. Human Reproduction, 23, 2359-2368. https://doi.org/10.1093/humrep/den260

[10] Clayton, H.B., Schieve, L.A., Peterson, H.B., Jamieson, D.J., Reynolds, M.A. and Wright, V.C. (2006) Ectopic Pregnancy Risk with Assisted Reproductive Technology Procedures. Obstetrics and Gynaecology, 107, 595-604. https://doi.org/10.1097/01.AOG.0000196503.78126.62

[11] Malak, M., Tawfeeq, T., Holzer, H. and Tulandi, T. (2011) Risk Factors for Ectopic Pregnancy after in Vitro Fertilization Treatment. Journal of Obstetrics and Gynaecology Canada, 33, 617-619. https://doi.org/10.1016/S1701-2163(16)34910-6

[12] Chang, H.J. and Suh, C.S. (2010) Ectopic Pregnancy after Assisted Reproductive Technology: What Are The Risk Factors? Current Opinion in Obstetrics \& Gynecology, 22, 202-207.

[13] Zhang, B., Cui, L., Tang, R., Ding, L., Yan, L. and Chen, Z.J. (2017) Reduced Ectopic Pregnancy Rate on Day 5 Embryo Transfer Compared with Day 3: A Meta-Analysis. PLoS ONE, 12, e0169837. https://doi.org/10.1371/journal.pone.0169837 